\title{
$\Delta 133 p 53$ represses p53-inducible senescence genes and enhances the generation of human induced pluripotent stem cells
}

\author{
Izumi Horikawa ${ }^{1}$, Kye-yoon Park ${ }^{2}$, Kazunobu Isogaya ${ }^{1}$, Yukiharu Hiyoshi ${ }^{1}$, Han Li ${ }^{3}$, Katsuhiro Anami ${ }^{1}$, Ana I Robles ${ }^{1}$, Abdul M Mondal ${ }^{1}$, \\ Kaori Fujita ${ }^{1}$, Manuel Serrano ${ }^{3}$ and Curtis C Harris ${ }^{*, 1}$
}

p53 functions to induce cellular senescence, which is incompatible with self-renewal of pluripotent stem cells such as induced pluripotent stem cells (iPSC) and embryonic stem cells (ESC). However, p53 also has essential roles in these cells through DNA damage repair for maintaining genomic integrity and high sensitivity to apoptosis for eliminating severely damaged cells. We hypothesized that $\Delta 133 \mathrm{p} 53$, a physiological inhibitory p53 isoform, is involved in the balanced regulation of self-renewing capacity, DNA damage repair and apoptosis. We examined 12 lines of human IPSC and their original fibroblasts, as well as three ESC lines, for endogenous protein levels of $\Delta 133$ p53 and full-length p53 (FL-p53), and mRNA levels of various p53 target genes. While FL-p53 levels in IPSC and ESC widely ranged from below to above those in the fibroblasts, all IPSC and ESC lines expressed elevated levels of $\Delta 133 p 53$. The $p 53$-inducible genes that mediate cellular senescence (p21 ${ }^{\text {WAF1 }}$, miR-34a, PAI-1 and IGFBP7), but not those for apoptosis (BAX and PUMA) and DNA damage repair (p53R2), were downregulated in iPSC and ESC. Consistent with these endogenous expression profiles, overexpression of $\Delta 133 \mathrm{p} 53$ in human fibroblasts preferentially repressed the p53-inducible senescence mediators and significantly enhanced their reprogramming to iPSC. The iPSC lines derived from $\Delta 133 p 53-$ overexpressing fibroblasts formed well-differentiated, benign teratomas in immunodeficient mice and had fewer numbers of somatic mutations than an iPSC derived from p53-knocked-down fibroblasts, suggesting that $\Delta 133 p 53$ overexpression is non- or less oncogenic and mutagenic than total inhibition of p53 activities. Overexpressed $\Delta 133 p 53$ prevented FL-p53 from binding to the regulatory regions of $\mathrm{p}^{\mathrm{WAF} 1}$ and miR-34a promoters, providing a mechanistic basis for its dominant-negative inhibition of a subset of p53 target genes. This study supports the hypothesis that upregulation of $\Delta 133 p 53$ is an endogenous mechanism that facilitates human somatic cells to become self-renewing pluripotent stem cells with maintained apoptotic and DNA repair activities. Cell Death and Differentiation (2017) 24, 1017-1028; doi:10.1038/cdd.2017.48; published online 31 March 2017

p53 regulates a variety of biological processes, including cellular senescence, apoptosis and DNA damage response. ${ }^{1-3}$ Cellular pluripotency and differentiation potential are critical to tissue homeostasis and regeneration and thus contribute to healthy lifespan in humans. ${ }^{4}$ p53 functions to regulate pluripotency and differentiation through the transcriptional regulation of its target genes. ${ }^{5,6}$ The ability of p53 to induce cellular senescence may be incompatible with the selfrenewing potential of iPSC and ESC, since p53 and cellular senescence act as a barrier to iPSC reprogramming in vitro in a cell-autonomous manner. ${ }^{7-11}$ Although $\mathrm{p} 16^{\text {INK4A }} / \mathrm{ARF}$ mediated cellular senescence promotes in vivo reprogramming through secretory cytokines, p53 still functions to limit reprogramming in vivo as well. ${ }^{12}$ On the other hand, the activity of p53 in DNA damage response and repair plays an essential role in maintaining genomic stability and preventing malignant transformation in iPSC and ESC. ${ }^{13,14}$ High rates of apoptosis in human iPSC and ESC ${ }^{15,16}$ contributes to elimination of damaged cells and is also regulated by p53. It is important to identify a regulator of p53 that possibly coordinates these different functions of p53 in human pluripotent stem cells.

The human TP53 gene encodes N-terminally or C-terminally truncated isoforms, in addition to the full-length p53 protein. ${ }^{17}$ Among those natural p53 isoforms, an N-terminally truncated $\Delta 133 p 53$ (which lacks the $\mathrm{N}$-terminal 132 amino acids) inhibits the activity of wild-type, full-length p53 (FL-p53). ${ }^{17-20}$ Unlike FL-p53 that is subject to proteasomemediated degradation, $\Delta 133 p 53$ is degraded via chaperoneassisted selective autophagy, ${ }^{21,22}$ which leads to its downregulation during replicative cellular senescence in normal human fibroblasts, astrocytes and $\mathrm{CD} 8^{+} \mathrm{T}$ lymphocytes. ${ }^{18-20}$ The specific knockdown of $\Delta 133 p 53$, mimicking its senescence-associated downregulation, relieves FL-p53 from inhibition by this isoform and results in the induction of cellular senescence in these normal human cells. ${ }^{18-20}$ Conversely, the overexpression of $\Delta 133 p 53$ delays the onset of replicative cellular senescence and extends the replicative lifespan, while it does not lead to cellular immortalization or malignant transformation by itself. ${ }^{18,19}$ It should also be noted that

\footnotetext{
${ }^{1}$ Laboratory of Human Carcinogenesis, Center for Cancer Research, National Cancer Institute, National Institutes of Health, Bethesda, MD 20892, USA; ${ }^{2} \mathrm{NIH}$ Stem Cell Unit, National Institutes of Health, Bethesda, MD 20892, USA and ${ }^{3}$ Tumor Suppression Group, Spanish National Cancer Research Center, Madrid 28029, Spain ${ }^{*}$ Corresponding author: CC Harris, Laboratory of Human Carcinogenesis, Center for Cancer Research, National Cancer Institute, National Institutes of Health, 37 Convent Drive, Room 3068A, Bethesda, MD 20892, USA. Tel: 301496 2048; Fax: 301496 0497; E-mail: harrisc@mail.nih.gov

Received 17.8.16; revised 27.2.17; accepted 01.3.17; Edited by X Lu; published online 31.3.2017
} 
$\Delta 133 p 53$ is likely to exist only in humans and primates, since any other organisms examined, including mice, do not have a methionine codon at the amino acid position corresponding to human codon 133 (ref. 20). These characteristics of $\Delta 133 p 53$ prompted us to hypothesize that the expression of this p53 isoform may play a unique role in human pluripotent stem cells. In this study we show expression, functional and genetic data supporting this hypothesis.

\section{Results}

Human pluripotent stem cells express abundant levels of endogenous $\Delta 133 p 53$ protein. We first investigated the expression levels of endogenous FL-p53 and $\Delta 133 p 53$ protein in human pluripotent stem cells. Twelve lines of human iPSC (named i14 through i25), a normal human fibroblast strain (CRL-2097) from which all these iPSC lines were derived, and 3 human ESC lines (WA01, WA07 and WA09) were examined in western blot analysis (Figure 1). The pluripotent status of these human iPSC and ESC lines was confirmed by the expression of Oct-4 (Figure 1) and Nanog (Supplementary Figure S1a). The expression levels of FL-p53 protein widely varied among these pluripotent stem cell lines, ranging from below (e.g., i17, i22, i23 and the three ESC lines) to above (e.g., i18, i21, i24 and i25) the level in CRL-2097 fibroblasts (Figure 1), which may be associated with two distinct p53-related states of human pluripotent stem cells. ${ }^{23}$ In contrast, all the iPSC and ESC lines were revealed to express elevated levels of $\Delta 133 p 53$ protein, which were at least 10-fold higher than the level in CRL-2097 (Figure 1). The expression levels of $\Delta 133 p 53$ mRNA were 1.9-3.6-fold higher in iPSC and ESC than in CRL-2097, but to a lesser degree compared with the protein levels (Supplementary Figure S1b). Inhibition of autophagy by treatment with bafilomycin A1 in an iPSC line only slightly increased $\Delta 133 p 53$ protein, in contrast to marked stabilization of another autophagy substrate p62/SQSTM1 (ref. 22; Supplementary Figure S1c), suggesting that elevated expression of $\Delta 133 p 53$ in human pluripotent stem cells is attributed in part to decreased degradation of this selective autophagy substrate. When CRL-2097 transduced with the retroviral vector of the Yamanaka factors (i.e., Oct-4, Sox-2, Klf-4 and c-Myc) was examined for $\Delta 133 p 53$ protein expression prior to the iPSC reprogramming protocol, these factors by themselves had only a minimal effect on $\Delta 133 p 53$ (Supplementary Figure S1d), suggesting that the upregulation of endogenous $\Delta 133 p 53$ occurs largely during the reprogramming process.

A subset, but not all, of p53-inducible genes are repressed in human pluripotent stem cells. To investigate the transcriptional activity of p53 in human pluripotent stem cells, we next examined the mRNA expression levels of p53inducible genes of different functions in the above set of human iPSC, ESC and fibroblasts (Figure 2). Although each of the p53-inducible genes could be involved in multiple functions of $p 53$, four of the genes examined (p21 ${ }^{\text {WAF1 }}$, PAI-1, IGFBP7 and miR-34a) are reported to mediate p53-induced cellular senescence, ${ }^{1,24-27}$ while BAX and PUMA regulate apoptosis ${ }^{1,28,29}$ and p53R2 is involved in nuclear and mitochondrial DNA repair and homeostasis. ${ }^{1,30,31}$ All of the four p53-inducible genes mediating cellular senescence were significantly repressed in all the iPSC and ESC lines compared with CRL-2097 fibroblasts (Figure 2a-d), although the repression of miR-34a in two iPSC (i14 and i18) was less clear than the others (Figure 2d). The mRNA expression levels of BAX (Figure 2e) and PUMA (Figure 2f) were higher in all iPSC and ESC but one (WA09), which expressed similar levels to CRL-2097. All the iPSC and ESC lines showed elevated levels of mRNA expression of p53R2 (Figure $2 \mathrm{~g}$ ) compared with CRL-2097. These results indicate that human pluripotent stem cells are associated with the repression of a subset of p53-inducible genes, which are involved in the induction of cellular senescence.

Overexpression of $\Delta 133 p 53$ in normal human fibroblasts preferentially represses p53-inducible genes involved in cellular senescence. We reproduced an iPSC/ESC-like upregulation of $\Delta 133 p 53$ in normal human fibroblasts (BJ strain) by retrovirally overexpressing $\Delta 133 p 53$ (Figure $3 a$ ). As a control, shRNA (short-hairpin RNA) -mediated knockdown of p53 was also performed (Figure 3a). We examined mRNA expression levels of the above seven p53-inducible genes in

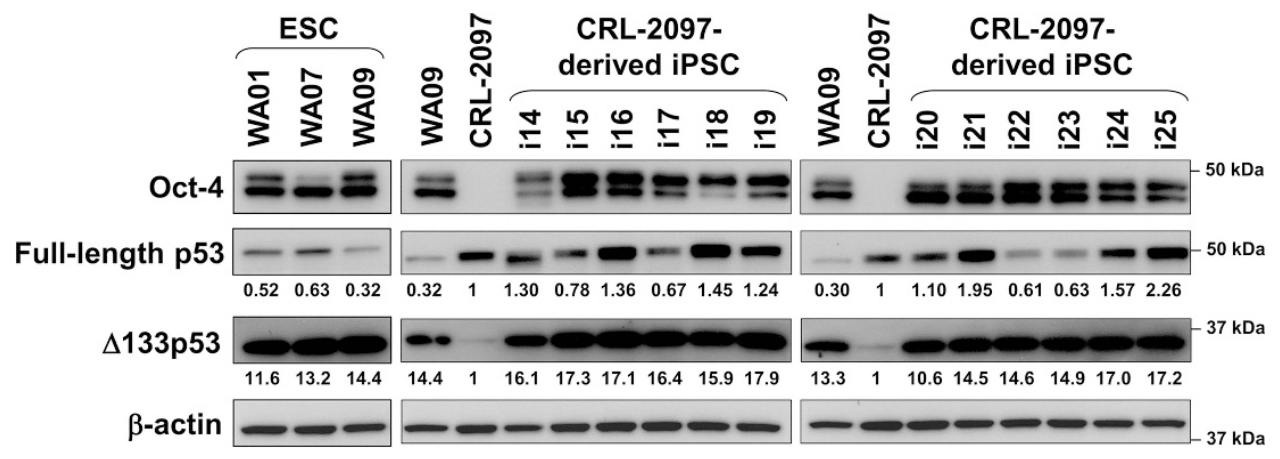

Figure 1 Human pluripotent stem cells express elevated levels of $\Delta 133 p 53$. Western blot analysis was performed using protein lysates prepared from a normal human fibroblast strain CRL-2097, 12 iPSC lines derived from CRL-2097 (i14 through i25) and three human ESC lines (WA01, WA07 and WA09). Oct-4 was a pluripotency marker positive in all IPSC and ESC lines. Full-length 553 was detected using the DO-1 antibody and $\Delta 133 p 53$ using the MAP4 antibody. ${ }^{18-21} \beta$-actin was a loading control for normalization in the quantitative image analysis. Results of three separate blots processed in parallel are shown, all of which contain WA09 and two of which (middle and right) contain CRL-2097 for cross-comparison. The expression levels of full-length p53 and $\Delta 133 p 53$ in iPSC and ESC lines are shown in relative to those in CRL-2097 (defined as 1.0) 
a

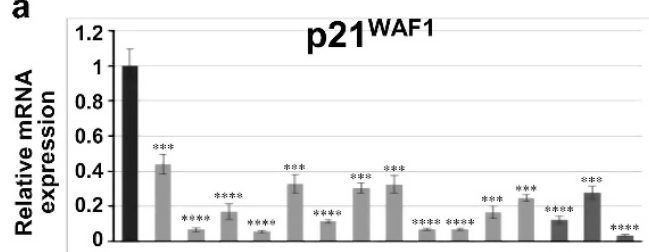

b

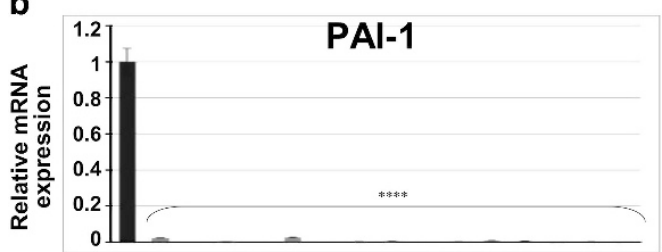

C

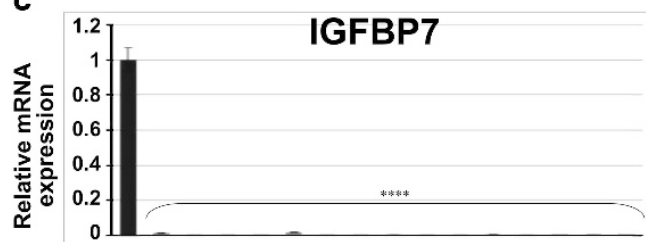

d

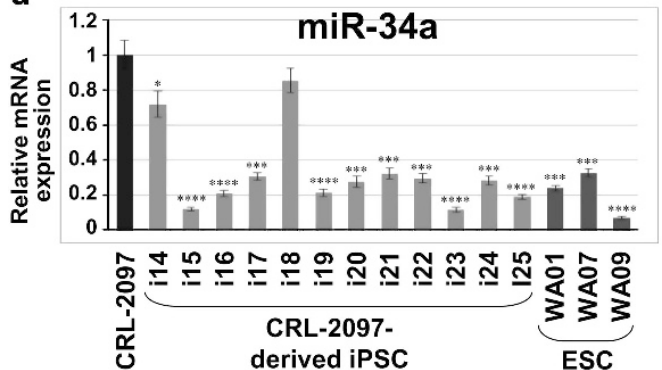

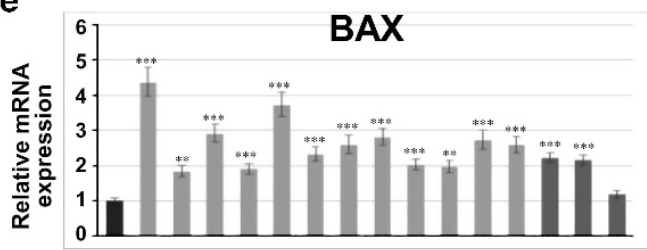

f

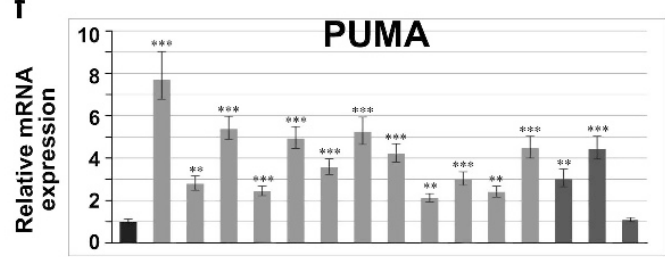

g

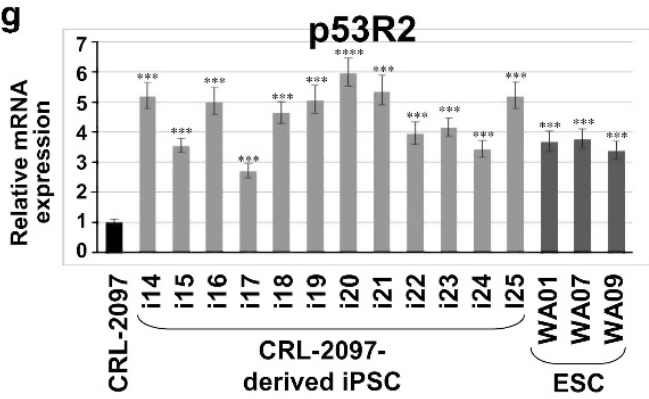

Figure 2 A subset of p53-inducible genes are repressed in human pluripotent stem cells. qRT-PCR analyses of mRNA expression of p21 WAF1 (a), PAl-1 (plasminogen activator inhibitor-1) (b), IGFBP7 (insulin-like growth factor binding protein 7) (c), miR-34a (microRNA-34a) (d), BAX (Bcl-2-associated X protein) (e), PUMA (p53 upregulated modulator of apoptosis) (f) and p53R2 (p53 inducible ribonucleotide reductase) (g) were performed in CRL-2097 human fibroblasts, their derived iPSC and human ESC (the same set of cells as in Figure 1; their names are below $\mathbf{d}$ and $\mathbf{g}$ ). Expression data are means \pm S.D. $(n=3)$ and shown in relative to $\mathrm{CRL}-2097$ (defined as 1.0). ${ }^{*} P<0.05,{ }^{* *} P<0.01$, ${ }^{* \star *} P<0.001,{ }^{* \star * \star} P<0.0001$, as determined by unpaired two-tailed Student's $t$-test

these $\Delta 133 p 53-o v e r e x p r e s s i n g ~ a n d ~ p 53-k n o c k e d-d o w n$ fibroblasts, along with their controls. All the seven p53-inducible genes were remarkably downregulated by p53 knockdown as expected (Figure $3 \mathrm{~b}$, left bars). The overexpression of $\triangle 133 p 53$ repressed p2 ${ }^{\text {WAF1 }}$, PAI-1, IGFBP7 and miR-34a, but not BAX, PUMA and p53R2 (Figure 3b, right bars). When $\triangle 133 p 53$ was overexpressed in CRL-2097 fibroblasts (Supplementary Figure S2a), similar results were obtained: p21 ${ }^{\mathrm{WAF} 1}$, PAI-1, IGFBP7 and miR-34a were repressed, while the other three genes were either unchanged (PUMA) or modestly downregulated (BAX and p53R2) (Supplementary Figure $S 2 b)$. These results suggest that $\Delta 133 p 53$ overexpression preferentially represses a subset of p53-inducible genes mediating cellular senescence in normal human fibroblasts, being coincident with the expression profiles in iPSC and ESC. To examine whether the three less responsive genes (BAX, PUMA and p53R2) are still activated by $p 53$ in the presence of abundant levels of $\Delta 133 p 53$, we transfected a siRNA targeting the $\mathrm{N}$-terminal region of $\mathrm{p} 53$ (therefore knocking down FL-p53 but not $\Delta 133 p 53$ ) to BJ and CRL-2097 fibroblasts with overexpressed $\Delta 133 p 53$ and to an iPSC line (Supplementary Figure S3a). We found that the expression of these three genes in these cells was largely or at least in part dependent on FL-p53 levels (Supplementary Figure S3b-d).

Overexpression of $\Delta 133 p 53$ enhances reprogramming from normal human fibroblasts to iPSC. Since $\Delta 133 p 53$ overexpression in normal human fibroblasts reproduced the repression of the p53-inducible senescence-mediating genes observed in human pluripotent stem cells (Figure 3 and Supplementary Figure S2), we examined whether it also enhances reprogramming from normal human fibroblasts to iPSC. BJ fibroblasts with and without overexpressed $\Delta 133 p 53$, along with those with p53 knockdown as a positive control (the same cells as in Figure 3), were used in an iPSC reprogramming protocol with retroviral transduction of three (Oct-4, Sox-2 and KIf-4; Figure 4a) or four Yamanaka factors 
a

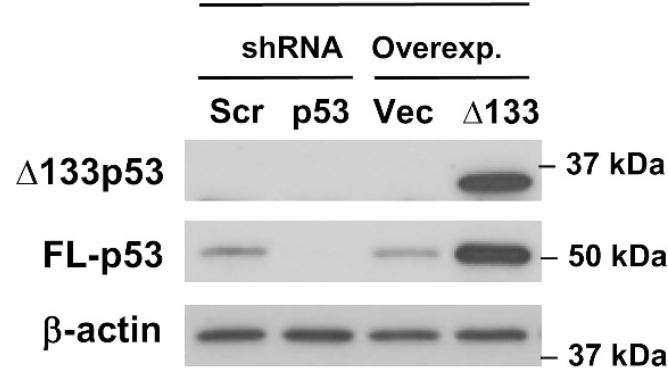

b
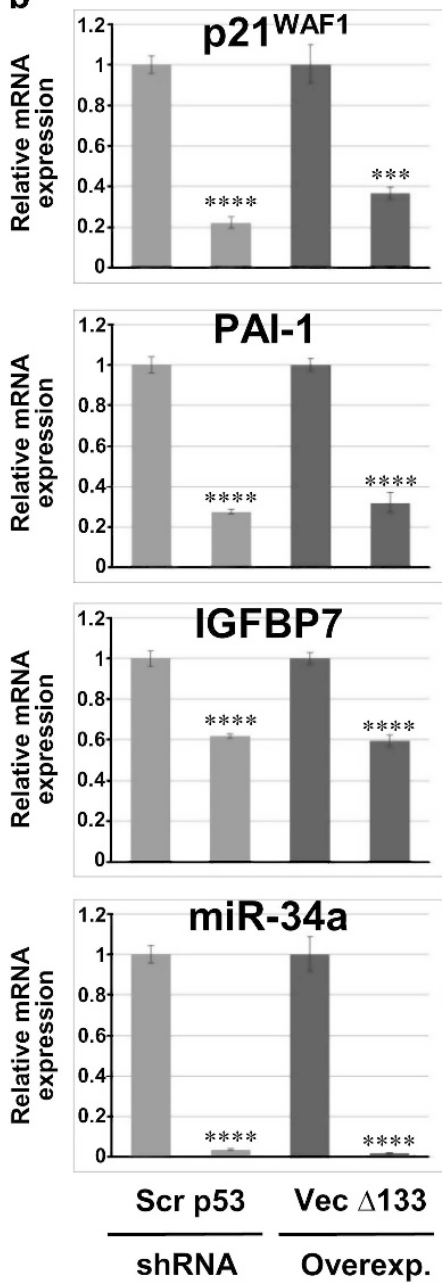
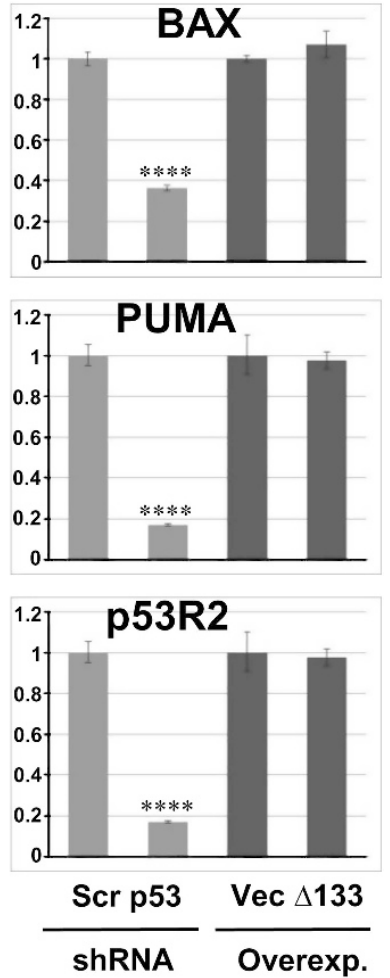

Figure 3 Overexpression of $\Delta 133 p 53$ in human fibroblasts represses a subset of p53-inducible genes. Normal human fibroblasts (BJ strain) were retrovirally transduced with an shRNA knockdown vector against p53 and its scrambled control (Scr), as well as with an overexpression vector of $\Delta 133 p 53$ and its empty vector control (Vec). (a) Western blot analysis confirming p53 knockdown and $\Delta 133 p 53$ overexpression. $\Delta 133 p 53$, full-length $p 53$ (FL-p53) and $\beta$-actin were detected as in Figure 1. $\Delta 133 p 53$ overexpression also increased FL-p53 as in previous publications. ${ }^{40}$ (b) mRNA expression of p53-inducible genes. The qRT-PCR analyses were performed as in Figure 2. The expression levels in p53-knocked-down and $\Delta 133$ p53-overexpressing cells are shown in relative to those in Scr and Vec controls (each defined as 1.0), respectively. Means \pm S.D. are shown, $n=3$. ${ }^{* * *} P<0.001,{ }^{* * *} P<0.0001$, as determined by unpaired two-tailed Student's $t$-test

(the former three plus C-Myc; Figure $4 b$ ). ${ }^{32}$ In both cases, significantly increased numbers of iPSC colonies were generated from the $\Delta 133 p 53$-overexpressing fibroblasts compared with the vector control cells, although to a lesser degree than from p53-knocked-down cells (Figure 4a and b). We also used another pair of normal human fibroblasts, CRL-2097 with and without overexpressed $\Delta 133 p 53$ (the same cells as in Supplementary Figure S2), in a separate reprogramming protocol based on transfection of synthetic mRNA for the four Yamanaka factors, ${ }^{33}$ which again showed that $\Delta 133 p 53$ overexpression increased the frequency of generation of iPSC colonies (Figure $4 \mathrm{c}$ and Supplementary Figure S4a). The mRNA transfection-based protocol was also carried out using BJ fibroblasts with inducible $\Delta 133 p 53$ expression (Supplementary Figure S4b), and the induction of $\Delta 133$ p53 was shown to enhance iPSC reprogramming (Figure 4d).
iPSC lines generated from $\Delta 133 p 53$-overexpressing fibroblasts form benign teratomas, have normal chromosomes and stable microsatellite repeats, and are similar to original fibroblasts or other iPSC lines in mitochondrial DNA copy number. We isolated and established iPSC lines that were generated from $\Delta 133 p 53$-overexpressing, p53-knocked-down, control vector-transduced and untransduced fibroblasts (all cell lines established in this study are listed in Supplementary Table S1). In a teratoma formation

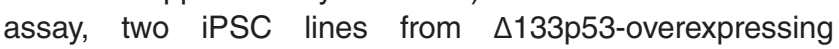
CRL-2097 fibroblasts (Ci133-3 and Ci133-5), along with two iPSC lines from control vector-transduced CRL-2097 (CiV1 and CiV4) and one from untransduced CRL-2097 (CiC1), were subcutaneously injected into NOD/SCID mice. All of the five iPSC lines formed teratomas with no malignant histology and with all three germ layer-derived tissues (Supplementary Table S1 and Figure 4e). The teratomas from one $\Delta 133 p 53$ 
a

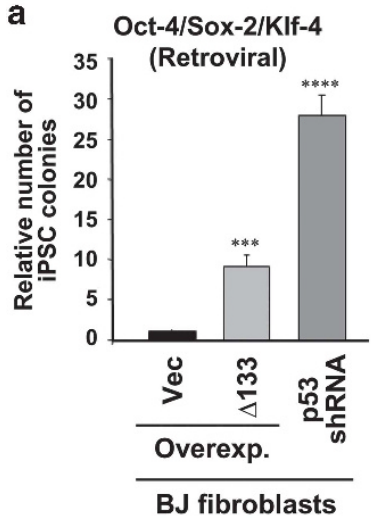

b

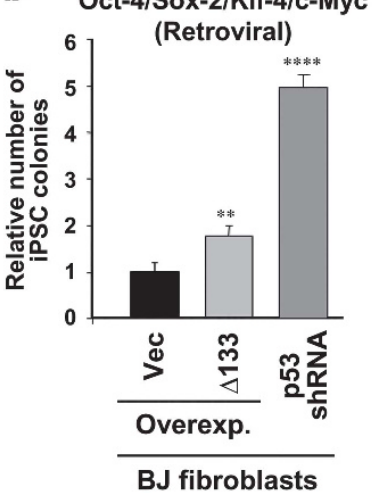

c

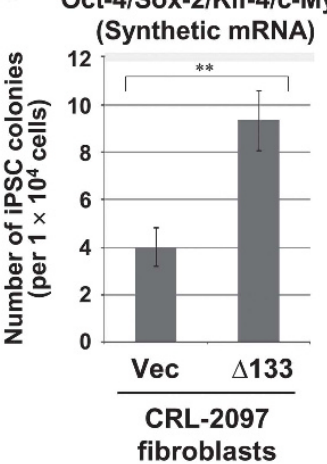

d

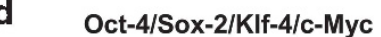

(Synthetic mRNA)

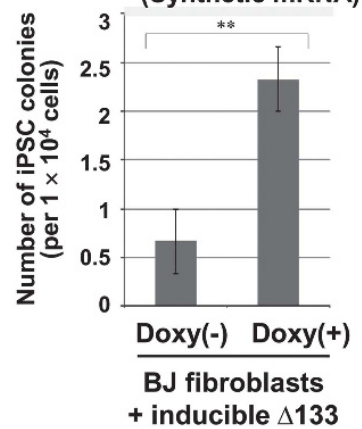

e

$$
\text { Ectoderm }
$$

\begin{tabular}{ccc}
\multicolumn{3}{c}{ Mesoderm } \\
\hline Cartilage & $\begin{array}{c}\text { Adipose } \\
\text { Tissue }\end{array}$ & $\begin{array}{c}\text { Smooth } \\
\text { Muscle }\end{array}$
\end{tabular}

\begin{tabular}{cc} 
Endoderm \\
\hline Gut $\quad \begin{array}{c}\text { Respiratory } \\
\text { Epithelium }\end{array}$
\end{tabular}
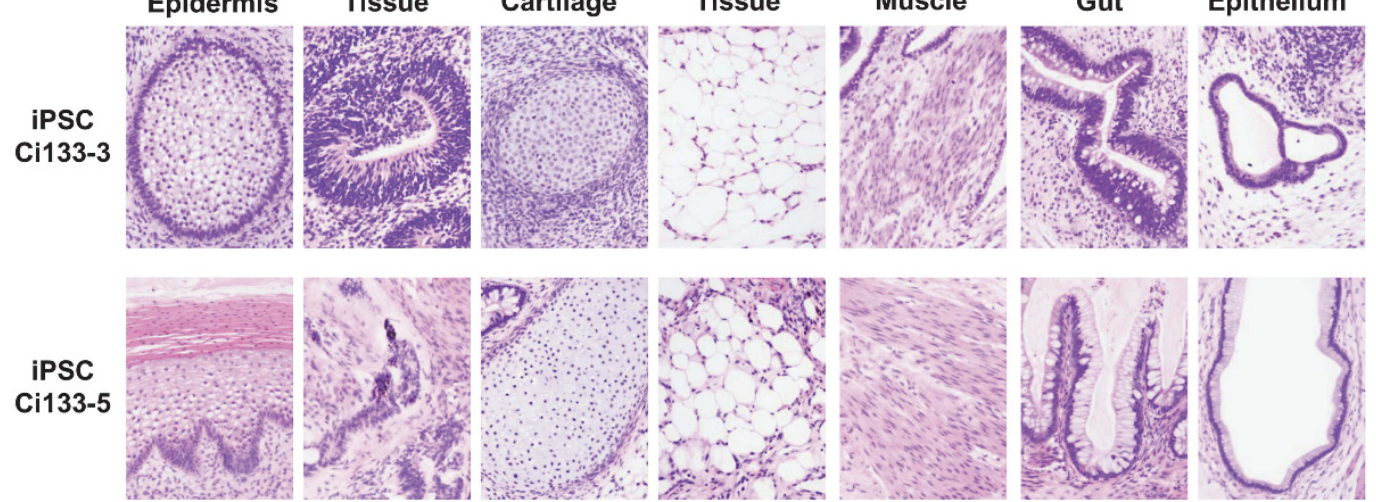

Figure 4 Overexpression of $\Delta 133 p 53$ enhances reprogramming from human fibroblasts to iPSC. (a and $\mathbf{b})$ BJ human fibroblasts with overexpressed $\Delta 133 p 53$ and the control vector (Vec) (those examined in Figure 3) were used for generating IPSC by retrovirally expressing three reprogramming factors (Oct-4, Sox-2 and Klf-4) (a) or four reprogramming factors (the former three plus c-Myc) (b). BJ human fibroblasts with shRNA knockdown of p53 (also examined in Figure 3) were included as a positive control. The

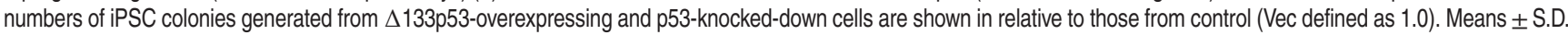
are shown, $n=3$. (c) CRL-2097 human fibroblasts with overexpressed $\Delta 133 p 53$ and the control vector (Vec) (those examined in Supplementary Figure S2) were used for generating iPSC by transfection of synthetic mRNAs encoding the four reprogramming factors. The graphs show the numbers of iPSC colonies generated per $1 \times 10^{4}$ cells. Means \pm S.D. are shown, $n=3$. A representative image is shown in Supplementary Figure S4a. (d) BJ human fibroblasts were transduced with a Tet repressor vector and then with a lentiviral vector for inducible expression of $\Delta 133 \mathrm{p} 53$. The inducible expression by treatment with doxycycline was confirmed by western blot (as shown in Supplementary Figure S4b). The cells in the presence (+) or absence $(-)$ of doxycycline (Doxy) were used in synthetic mRNA-mediated iPSC reprogramming as performed in c. Data are also presented as in c. Means \pm S.D. are shown, $n=3 .{ }^{\star \star} P<0.01,{ }^{\star \star \star} P<0.001,{ }^{\star \star \star \star} P<0.0001$, as determined by unpaired two-tailed Student's $t$-test. (e) Teratoma formation assay showing in vivo differentiation of iPSC generated from $\Delta 133$ p53-overexpressing cells. Two iPSC lines generated from CRL-2097 fibroblasts overexpressing $\Delta 133 p 53$ (Ci133-3 and Ci133-5; isolated and expanded from the experiment shown in c) were subcutaneously injected into NOD/SCID mice. Teratomas were resected after 7 weeks (Ci133-3) or 21 weeks (Ci133-5), followed by formalin fixation and paraffin embedding, and then hematoxylin-eosin staining of tissue sections. Differentiated tissues derived from all three germ layers are shown

overexpression-derived iPSC (Ci133-5) were more maturely differentiated (Figure $4 \mathrm{e}$, lower panels) than those from the other four lines for currently unknown reason.

Six iPSC lines generated from $\Delta 133 p 53$-overexpressing fibroblasts (Bi133-1, Bi133-3, Ci133-2, Ci133-3, Ci133-4 and Ci133-5), two lines from control or untransduced fibroblasts (CiC1 and $\mathrm{CiV} 2)$ and an iPSC line from p53-knocked-down fibroblasts (Bi53KD) were examined by G-banding chromosome analysis, and all of them were found to have normal karyotype (46, XY) with no gross chromosomal aberrations (Supplementary Table S1 and Supplementary Figure S4c). When five highly variable microsatellite loci (ACTC, BAT26, D5S107, D5S406 and D13S153) ${ }^{34}$ were examined, all iPSC lines had the identical allelic profiles to their original fibroblasts BJ or CRL-2097 at the all five loci (Supplementary Table
S2 and Supplementary Figure S5), suggesting that these microsatellite repeats were stably maintained and confirming that all the iPSC lines established in this study were indeed derived from their original fibroblasts. The copy numbers of mitochondrial DNA (mtDNA) were different from cell line to cell line, ranging from 0.9- to 3.3-fold of the original fibroblasts, and there was not a consistent change associated with iPSC lines from $\Delta 133 p 53-o v e r e x p r e s s i n g$ fibroblasts (Supplementary Figure S6). A mtDNA D-loop region, which was previously reported to contain homoplasmic and heteroplasmic mutations in $\mathrm{iPSC},{ }^{35}$ was sequenced and there was no mutation in any of the iPSC lines established in this study, although the BJ- and CRL-2097-specific polymorphisms again confirmed the origin of each iPSC line (Supplementary Table S2). 
iPSC lines generated from $\Delta 133 p 53-o v e r e x p r e s s i n g$ fibroblasts, as well as control iPSC lines, have lower rates of somatic mutations than an iPSC line generated from p53-knocked-down fibroblasts. For precise and genome-wide examination of genetic changes in iPSC, we performed whole exome sequencing (Supplementary Table S3) and analyzed the data for somatic changes between each iPSC line examined and its original fibroblasts. As predicted from the total inhibition of p53 activities, an iPSC from p53-knocked-down fibroblasts (Bi53KD) had the highest rate of somatic mutations (5.1 mutations per $\mathrm{Mb}$ ), including synonymous and nonsynonymous single-nucleotide variants (SNV) and small insertions and deletions (InDel) (Figure 5a). Four iPSC lines from $\triangle 133 p 53-o v e r e x p r e s s i n g$ fibroblasts had 1.2 (Bi133-1), 0.5 (Bi133-3), 0.8 (Ci133-2) and 0.2 (Ci133-3) somatic mutations per $\mathrm{Mb}$, while the mutation rates in four control iPSC lines were 0.4 (BiC1), 1.8 [Bi133(-)], 0.1 (CiV2) and 0.9 (CiV4), indicating that these two groups of iPSC lines have similar rates of somatic mutations (Figure 5a). When somatic mutations affecting amino acid sequences in protein-coding genes (i.e., missense, premature stop and frameshift mutations) were compared, Bi53KD again had the most mutations (Figure 5b and Supplementary Table S4). Although the numbers of such coding mutations in the other iPSC lines widely varied from 4 to 20 , there was no association with whether or not they were generated from $\Delta 133 p 53-o v e r e x p r e s s i n g$ fibroblasts (Figure $5 \mathrm{~b}$ ). These results using a mutant allele frequency cutoff of 0.30 are roughly consistent with the previous studies that reported an average of 6 or 12 somatic coding mutations in homozygous or heterozygous state in iPSC lines. ${ }^{36,37}$ Out of 116 genes we identified as carrying somatic coding mutations, 114 genes were unique to a single iPSC line (Supplementary Table S4), strongly supporting that each iPSC line is an independent clone.

iPSC lines generated from $\Delta 133 p 53-o v e r e x p r e s s i n g$ fibroblasts and control iPSC lines both express similar levels of $\Delta 133 p 53$ protein and p53 target genes. As above, although $\triangle 133 p 53$ overexpression enhances iPSC generation, the iPSC lines we established have similar biological and genetic characteristics whether or not their original fibroblasts had $\Delta 133 p 53$ overexpression. We thus examined whether they are also similar in expression of $\Delta 133 p 53$ protein and p53 target genes. Both $\Delta 133 p 53$ overexpression-enhanced (Ci133-1, Ci133-2, Ci133-3 and Ci133-4) and control iPSC lines (CiV1, CiV2 and CiV4) were found to express similar ranges of $\Delta 133 p 53$ protein, whether exogenous or endogenous (Supplementary Figure S7a), which are also similar to those in previously established iPSC and ESC lines (Figure 1). The expression levels of the p53 target genes in these iPSC lines (Supplementary Figure $\mathrm{S} 7 \mathrm{~b}-\mathrm{h}$ ) also reproduced the results in previously established iPSC and ESC lines (Figure 2) and there was no consistent difference between $\Delta 133 p 53$ overexpression-enhanced and control iPSC lines in any of the genes examined. These results suggest that both exogenous overexpression and endogenous upregulation of $\Delta 133 p 53$ similarly contribute to iPSC reprogramming, although at an increased efficiency with the former.
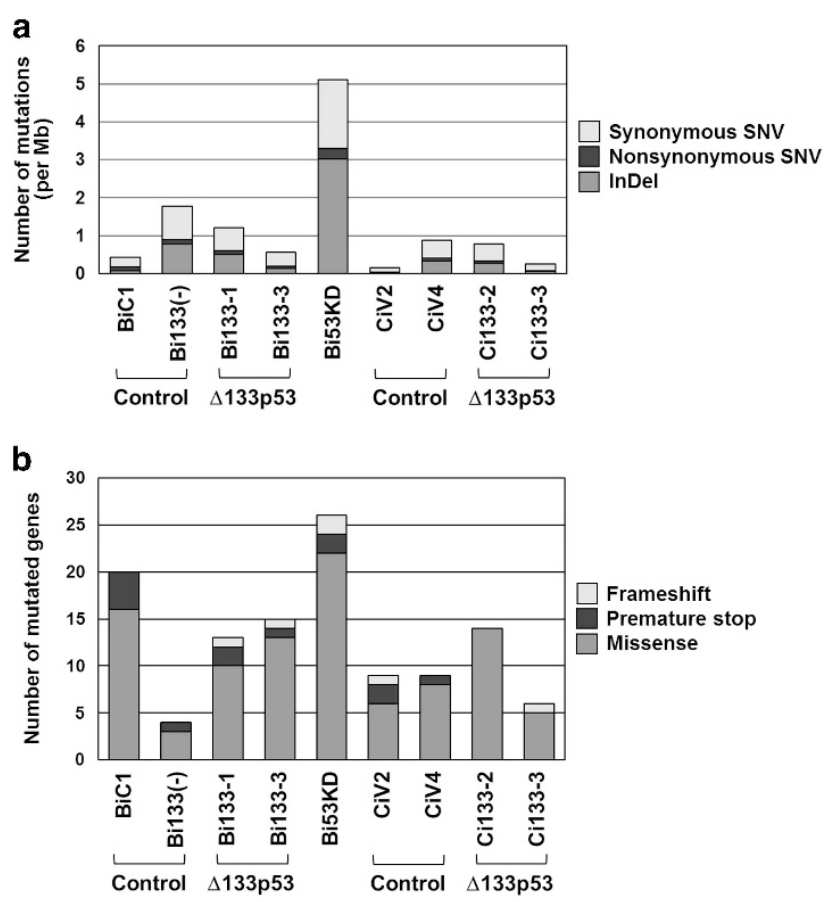

Figure 5 Summary of whole-exome sequencing data in iPSC lines. (a) Numbers of somatic mutations per megabase (Mb), including synonymous and nonsynonymous single-nucleotide changes (SNV) and small insertions and deletions (InDel). The iPSC lines derived from $\Delta 133 p 53$-overexpressing fibroblasts $(\Delta 133 p 53)$ and those from fibroblasts without $\Delta 133 p 53$ overexpression (control) are indicated. (b) Numbers of protein-coding genes that have a missense, premature stop or frameshift mutation with mutant allele frequency of 0.30 or greater. The genes mutated in each iPSC line are listed in Supplementary Table S4

Overexpressed $\Delta 133 p 53$ displaces full-length p53 from the promoter regions of the p53-inducible genes. Consistent with the retention of the C-terminal oligomerization domain (Figure 6a), $\Delta 133 p 53$ was shown to physically interact with FL-p53 (Figure $6 \mathrm{~b}$ ) as previously reported. ${ }^{21}$ To examine how $\Delta 133 p 53$ affects the transactivation function of FL-p53 and to gain mechanistic insight into its role in enhancing iPSC reprogramming, we performed a chromatin immunoprecipitation (ChIP) assay for binding of FL-p53 to the p53 response elements within the regulatory regions of the miR-34a, p21 WAF1, BAX and PUMA genes (Figure 6a). Since the DO-1 antibody used in this assay recognizes the $\mathrm{N}$-terminal amino acid residues $20-25$ of FL-p53 (Figure 6a), any $\mathrm{N}$-terminally truncated p53 isoforms (including $\Delta 133 p 53$ ) by themselves could not be immunoprecipitated. Moreover, the major pattern of $\mathrm{C}$-terminal alternative mRNA splicing in proliferating human fibroblasts was found to be a-splicing encoding FL-p53, with no or minor fractions of $\beta$-and $\gamma$-splicing patterns leading to C-terminal truncations. ${ }^{18,38}$ Thus, this ChIP assay was designed to exclusively or at least mainly detect DNA binding complexes involving FL-p53. We also confirmed that overexpressed $\Delta 133 p 53$ did not interfere with the ability of DO-1 to immunoprecipitate FL-p53 (Figure 6b). While no binding to the negative control region $(H B B)$ was observed in immortalized human fibroblasts with or without $\triangle 133 p 53$ overexpression, the miR-34a, p21 WAF1, $B A X$ and $P U M A$ promoter regions showed significant binding 


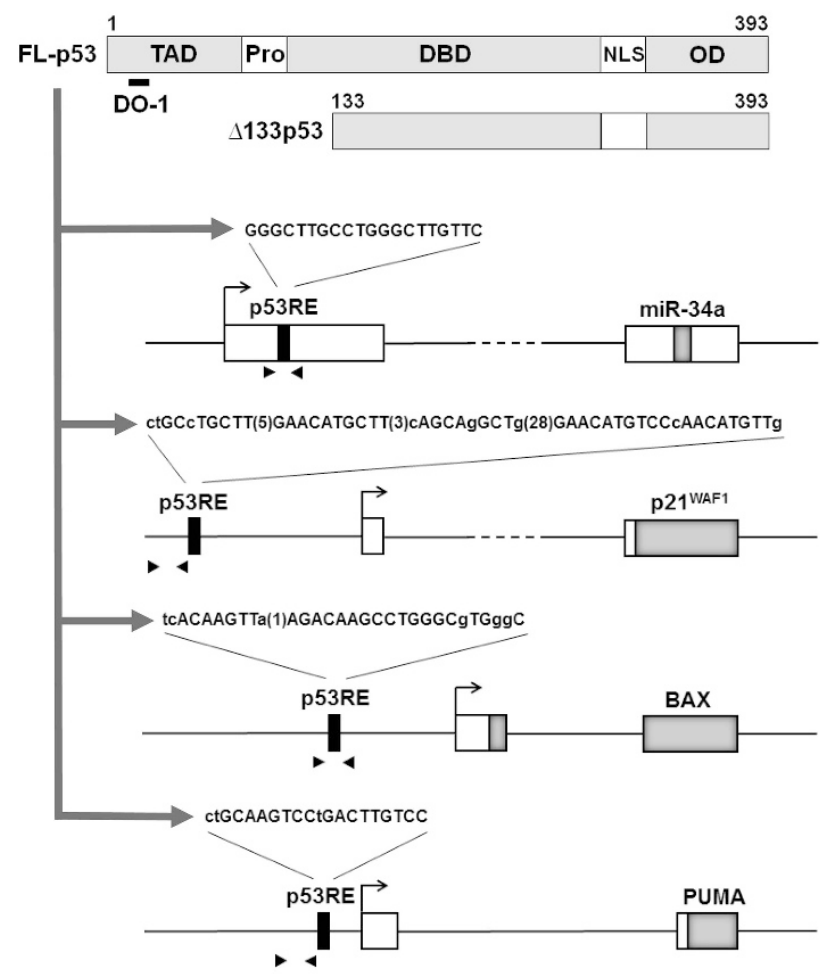

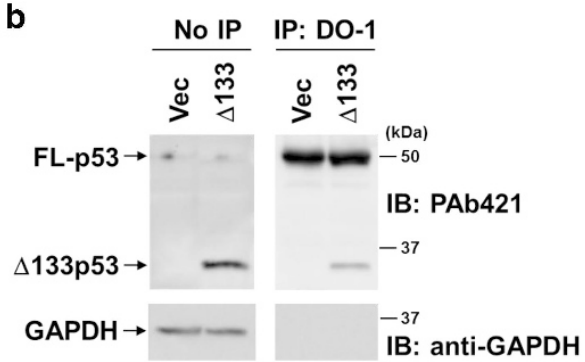

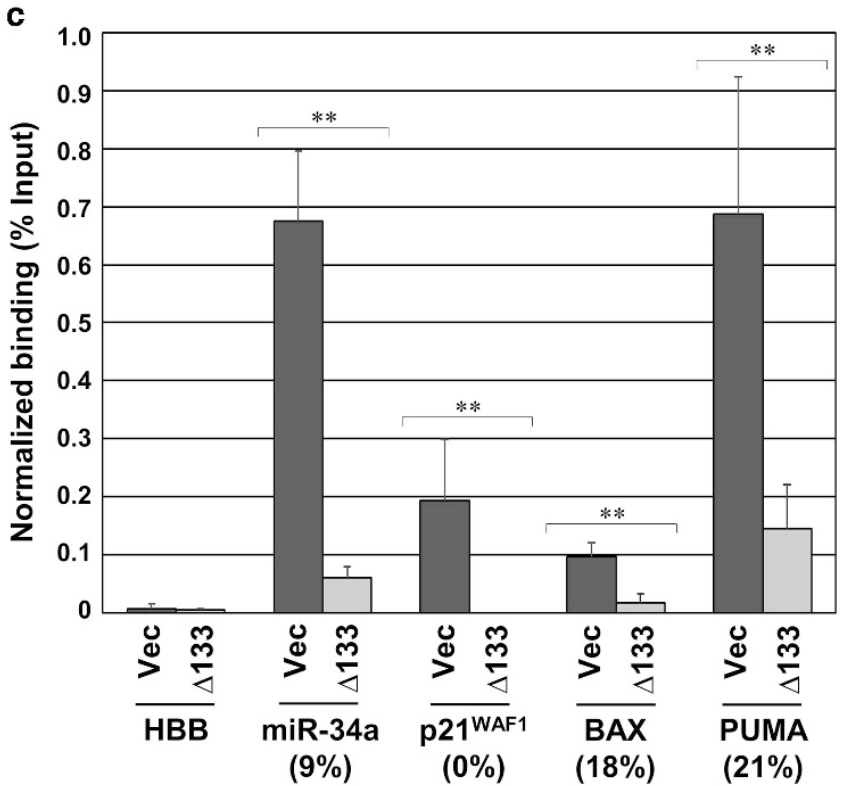

(Relative binding with overexpressed $\Delta 133 p 53$ )

Figure 6 Overexpressed $\triangle 133 p 53$ displaces full-length p53 from the p53-inducible gene promoters. (a) Chromatin immunoprecipitation (ChIP)-qPCR assay for p53 binding to the regulatory regions of the miR-34a, p2 $1^{\text {WAF1 }}, B A X$ and PUMA genes. Domain structures of full-length $p 53$ (FL-p53) and $\triangle 133 p 53$ are shown (TAD, transactivation domain; Pro, proline-rich region; DBD, DNA-binding domain; NLS, nuclear localization signal; and OD, oligomerization domain). Since the anti-p53 antibody used, DO-1, recognizes the $\mathrm{N}$-terminal amino acids 20-25, this assay detects DNA binding of protein complexes containing FL-p53, but not that of any N-terminally truncated p53 isoforms by themselves, including $\triangle 133 p 53$. Also shown are the positions and DNA sequences of p53 response elements (p53RE) that transcriptionally activate miR-34a, p21 ${ }^{\text {WAF1 }}$, BAX and PUMA. Upper and lowercase letters show matched and mismatched bases, respectively, to the canonical p53RE sequence (RRRCWWGYYY), with numbers of intervening bases in the parentheses. Arrows indicate the transcription start sites. Paired arrowheads indicate the regions amplified by qPCR. (b) Overexpression of $\Delta 133 p 53$ does not affect the efficiency of immunoprecipitation by the DO-1 antibody. Protein samples from immortalized BJ fibroblasts overexpressing $\Delta 133 p 53$ or with control vector (Vec), either after immunoprecipitation (IP) with DO-1 or directly (No IP), were used in western blot (IB) with PAb421, an antibody recognizing the C-terminus of p53, and with anti-GAPDH antibody (a loading control and a negative control for IP with DO-1). FL-p53 was immunoprecipitated by D0-1 at similar efficiencies in both cells. The interaction between FL-p53 and $\Delta 133$ p53 was also detected. (c) Quantitative data of FL-p53 binding to the p53RE. Crosslinked protein-DNA complexes from immortalized BJ fibroblasts overexpressing $\Delta 133$ p53 or with control vector (Vec) were analyzed by ChIP-qPCR as outlined in (a). HBB (hemoglobin subunit $\beta$ ) was used as a negative control. Normalized binding values are shown as \% input. The relative binding in the presence of overexpressed $\Delta 133$ p53 compared with its absence is shown in the parenthesis for each gene. Means \pm S.D. are shown, $n=3 .{ }^{*} P<0.01$, as determined by unpaired two-tailed Student's $t$-test

in the absence of overexpressed $\Delta 133 p 53$ (Figure $6 c, V e c$ ). The overexpression of $\Delta 133 p 53$ significantly reduced the FLp53 binding to these four promoter regions (Figure 6c, $\Delta 133$ ), suggesting that $\Delta 133 p 53$ is able to displace FL-p53 from the p53 response elements through its hetero-oligomerization with FL-p53. The residual fraction of FL-p53 binding in the presence of overexpressed $\triangle 133 p 53$ to the $B A X$ and PUMA promoters (approximately $20 \%$ of the binding in its absence) was larger than that to the miR-34a and $p 21^{\text {WAF1 }}$ promoters ( $9 \%$ and undetectable, respectively). It is currently unknown whether the residual binding of FL-p53 is due to remaining
FL-p53 homooligomers or newly formed heterooligomers of $\Delta 133 p 53$ and FL-p53 in a promoter context-specific manner.

\section{Discussion}

This study for the first time establishes the role of $\Delta 133 p 53$ in human pluripotent stem cells by showing that: (i) endogenous $\triangle 133 p 53$ is upregulated in human iPSC and ESC lines (Figure 1); (ii) a subset of p53-inducible genes that mediate cellular senescence are downregulated in human pluripotent stem cells (Figure 2); (iii) the same subset of p53-inducible 
genes are repressed by $\Delta 133 p 53$ overexpression in human fibroblasts (Figure 3 ); and (iv) $\Delta 133 p 53$ enhances reprogramming from human fibroblasts to iPSC (Figure 4). Our wholeexome sequencing data suggest that $\Delta 133 p 53$ overexpression, unlike p53 knockdown, does not increase mutation rates during iPSC reprogramming (Figure 5). This study also provides a mechanistic insight into the dominant-negative inhibition of p53-inducible senescence genes by $\Delta 133 p 53$ (Figures 6 and 7). Our findings represent significant progress in understanding the p53 isoform-mediated regulation of the p53 signalling network in human development and physiology, although our $\Delta 133 p 53$ knockdown method established for normal fibroblasts, $\mathrm{CD}^{+} \mathrm{T}$ lymphocytes and astrocytes ${ }^{18-20}$ failed to successfully knock down the abundant levels of endogenous $\triangle 133 p 53$ in iPSC and ESC (data not shown).

We previously showed that downregulation of $\Delta 133 p 53$ during replicative senescence in normal human cells occurs through protein degradation via chaperone-assisted selective autophagy, ${ }^{21}$ while others reported that upregulation of $\Delta 133 p 53$ in some cancer cell lines and Helicobacter pyloriinfected cells may involve transcriptional activation. ${ }^{39,40}$ Our findings (Figure 1 and Supplementary Figures S1b, c and d) suggest that both reduced autophagic degradation and increased mRNA expression during reprogramming events contribute to the elevated levels of $\Delta 133 p 53$ in human pluripotent stem cells, although the exact regulators of these processes remain to be identified.

The ability of $\Delta 133 p 53$ to repress the p53-inducible senescence-mediating genes (Figure 3 and Supplementary Figure S2) and to enhance iPSC reprogramming (Figure 4) is well consistent with the upregulation of endogenous $\Delta 133 p 53$ (Figure 1) and the repression of the same subset of genes in human pluripotent stem cells (Figure 2). Our data are also consistent with the previous reports that p21 ${ }^{\mathrm{WAF} 1}$ and miR-34a function to restrict reprogramming to iPSC. ${ }^{7,41-43}$ This study suggests that overexpressed $\Delta 133 p 53$, and likely endogenously upregulated $\Delta 133 p 53$ as well, dominant-negatively inhibits the transactivation function of FL-p53 on the p53inducible senescence genes (such as miR-34a and $p 21^{W A F 1}$ ), leading to the suppression of p53-mediated cellular senescence, to induce and maintain the self-renewing potential in human pluripotent stem cells (Figure 7). Our findings that $\triangle 133 p 53$ did not, or did to a lesser degree, affect the expression of BAX, PUMA and p53R2 (Figure 3 and Supplementary Figure S2) are reminiscent of a previous report by Rasmussen et al. ${ }^{43}$ that transient suppression of p53 enhances iPSC reprogramming without affecting apoptosis and DNA damage response. The maintained or elevated expression of these genes in human iPSC and ESC (Figure 2) likely contributes to high sensitivity to apoptosis for eliminating damaged cells ${ }^{16}$ and maintenance of genomic stability ${ }^{44}$ in these human pluripotent stem cells.

There are a number of precedents for the transcriptional regulation of a select subset of p53 target genes, including those inducing either apoptosis or cell cycle arrest and senescence. ${ }^{45-47}$ In this study, the preferential repression of p2 $1^{\text {WAF1 }}$ and miR-34a, but not BAX and PUMA, by $\triangle 133 p 53$ may not fully be explained by the FL-p53 binding to their promoter regions in the ChIP-qPCR assay. However, since the expression of BAX and PUMA in the presence of

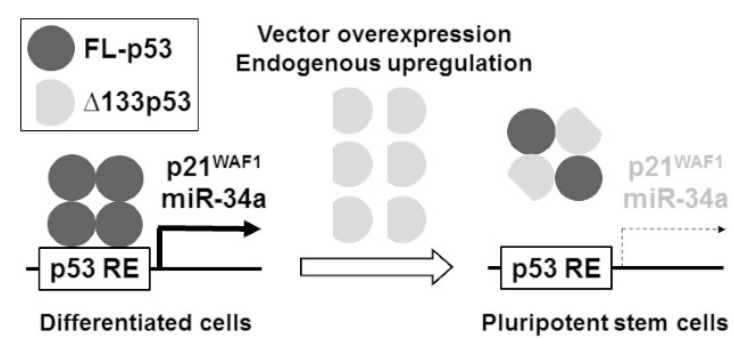

Figure 7 Schematic representation of the mechanism by which endogenously upregulated or exogenously overexpressed $\Delta 133 p 53$ contributes to the generation and maintenance of human pluripotent stem cells. $\Delta 133 p 53$ was previously shown to physically interact with FL-p53 (ref. 21), although the exact stoichiometry of the interaction is still unknown (a heterotetramer consisting of two each is shown in this scheme)

overexpressed $\Delta 133 p 53$ was still dependent on FL-p53 (Supplementary Figure S3) and the BAX and PUMA promoters showed the more fractions of residual binding of FL-p53 in the presence of overexpressed $\Delta 133 p 53$ (Figure $6 \mathrm{c}$ ), we speculate that $\Delta 133 p 53 / F L-p 53$ heterooligomers may bind to and activate these genes in a promoter context-dependent manner in human fibroblasts. Since $\Delta 133 p 53$ loses one amino acid making direct contact with DNA (Lys120) but retains all the other responsible amino acids, ${ }^{48}$ it is likely that such heterooligomers can still function as a DNA-binding transcriptional regulator but in a more context-dependent manner and/ or at a more limited set of genes. Our future studies will be aimed at determining the DNA binding profiles of $\Delta 133 p 53$ at a larger and genome-wide scale.

The tumorigenicity of iPSC is a serious safety concern ${ }^{14,49}$ and iPSC from p53-deficient or knocked-down cells were reported to become malignant and form teratocarcinoma in vivo. ${ }^{8,11}$ Our iPSC lines from $\Delta 133 p 53$-overexpressing fibroblasts, as well as control iPSC lines from fibroblasts without $\Delta 133 p 53$ overexpression, formed well-differentiated, benign teratomas with no malignant pathology (Figure $4 \mathrm{e}$ and Supplementary Table S1), rather than teratocarcinoma, indicating that $\Delta 133 p 53$ does not principally cooperate with the Yamanaka reprogramming factors towards malignant transformation. The whole exome sequencing analysis also revealed that the iPSC lines from $\Delta 133 p 53$-overexpressing fibroblasts, similar to control iPSC lines, showed lower somatic mutation rates and had fewer numbers of protein-coding mutations than an iPSC line from p53-knocked-down fibroblasts (Figure 5 and Supplementary Table S4). In addition, $\triangle 133 p 53$ overexpression-induced iPSC lines and control iPSC lines were found to be similar in eventual levels of $\Delta 133$ p53 protein, p53 target gene expression and genomic stability markers (Supplementary Figures S5, S6 and S7 and Supplementary Table S2). Our data suggest that the upregulation of $\Delta 133 p 53$ facilitates normal human somatic cells to be reprogrammed to a pluripotent stem cell state, and that overexpression of $\Delta 133 p 53$, unlike knockdown of p53, could be a non- or less oncogenic and mutagenic method to enhance the reprogramming process.

While this study highlights the role of $\Delta 133 p 53$ in human pluripotent stem cells through the regulation of p53-mediated cellular senescence, a more efficient iPSC reprogramming by 
p53 knockdown than by $\Delta 133 p 53$ overexpression (Figure $4 a$ and b) may suggest other p53-regulated processes (e.g., transient induction of apoptosis during reprogramming ${ }^{9,42}$ and regulation of energy metabolism ${ }^{1,50}$ ) as another barrier to reprogramming. Since this study and our previous publications ${ }^{18-21}$ place $\Delta 133 p 53$ at the crossroads of the regulation of autophagy, cellular senescence and pluripotent stem cells, it is interesting to investigate whether $\Delta 133 p 53$ also contributes to the activation of autophagy, which is associated with self-renewal of various types of stem cells and is required for efficient iPSC reprogramming. ${ }^{51}$ Our initial experiment to address this possibility showed that overexpression of $\Delta 133 p 53$ upregulates autophagy-mediating factors composing the core autophagy machinery, such as Beclin-1, ATG7 and ATG5-ATG12 conjugate, ${ }^{22}$ not only in normal human fibroblasts (Supplementary Figure S8a) but also in a p53-null fibroblast cell line (Supplementary Figure S8b). Further studies will elucidate whether $\Delta 133 p 53$-induced upregulation of these factors plays a complementary role in enhancing iPSC reprogramming via autophagy activation. These autophagy-mediating factors are also to be examined as possible targets of the transactivation function of $\Delta 133 p 53$ in a manner independent of FL-p53 (ref. 52).

\section{Materials and Methods}

Cells and cell culture. Normal human fibroblast strains derived from newborn foreskin (CRL-2097 and BJ) and fetal lung (MRC-5) were obtained from American Type Culture Collection (ATCC; Manassas, VA, USA). Immortalized BJ fibroblasts were generated by retroviral transduction of human telomerase reverse transcriptase (hTERT) ${ }^{53}$ MDAH041-/-, a p53-null fibroblast cell line with a homozygous frameshift mutation at codon 184 (refs 18,21), was provided by M. Tainsky (Case Western Reserve University). These fibroblasts were maintained in Dulbecco's modified Eagle medium (DMEM) supplemented with $10 \%$ fetal bovine serum. Human iPSC lines (i14 through i25) were derived from CRL-2097 by lentiviral transduction of the four Yamanaka factors at NIH Stem Cell Unit (http:// stemcells.nih.gov). ${ }^{54}$ Human ESC lines (WA01, WA07 and WA09) were also obtained from NIH Stem Cell Unit. These previously established IPSC and ESC lines were maintained feeder-free in mTeSR1 medium (STEMCELL Technologies, Vancouver, BC, Canada) on cell culture wells and plates coated with BD Matrigel hESC-qualified Matrix (BD Biosciences, San Jose, CA, USA). Harvesting of cells was carried out using ReLeSR (STEMCELL Technologies). Newly generated IPSC clones were initially expanded on mitomycin-C-treated SNL feeder cells (Cell Biolabs, San Diego, CA, USA) in Primate ES Cell Culture Medium supplemented with $4 \mathrm{ng} / \mathrm{ml}$ bFGF (ReproCELL, Yokohama, Japan) or in DMEM/F12 medium containing KnockOut Serum Replacement (KSR, 20\%; Thermo Fisher Scientific, Waltham, MA, USA), L-glutamine (2 mM), non-essential amino acids $\left(1 \times 10^{-4} \mathrm{M}\right)$, 2-mercaptoethanol $\left(1 \times 10^{-4} \mathrm{M}\right)$ and bFGF $(4 \mathrm{ng} / \mathrm{ml}),{ }^{32}$ and then transferred to feeder-free culture as above. Treatment of iPSC with bafilomycin A1 (Sigma-Aldrich, St. Louis, MO, USA) was at $100 \mathrm{nM}$ for $4 \mathrm{~h}$.

Protein lysate preparation and western blot analysis. Cells were lysed in $10 \mathrm{mM}$ Tris-HCl (pH 7.5)/150 mM NaCl/0.1\% SDS/1\% NP-40/1 mM EDTA containing Complete Protease Inhibitor Cocktail (Roche Diagnostics, Indianapolis, IN, USA). Protein lysates (20-40 $\mu$ g per sample) were separated on $10 \%$ or $4-20 \%$ gradient SDS-polyacrylamide gels (Thermo Fisher Scientific) and transferred to Hybond-P PVDF membranes (GE HealthCare Life Sciences, Pittsburgh, PA, USA). Incubations with primary and secondary antibodies were carried out in $10 \mathrm{mM}$ Tris$\mathrm{HCl}(\mathrm{pH} 7.5) / 150 \mathrm{mM} \mathrm{NaCl} / 0.05 \%$ Tween 20 containing 5\% non-fat dry milk (LabScientific, Highlands, NJ, USA) or SuperBlock (Thermo Fisher Scientific). Signal detection was performed using ECL western blotting detection system (GE HealthCare Life Sciences) or SuperSignal West Dura chemiluminescence substrate (Thermo Fisher Scientific). Quantitative image analysis was performed using the ImageJ $1.40 \mathrm{~g}$ software (http://rsb.info.nih.gov/ij/). The anti- $\Delta 133 \mathrm{p} 53$ antibody (MAP4, rabbit polyclonal; $1: 5000$ ) was used by Fujita et al.., ${ }^{18}$ Mondal et al.. ${ }^{19}$
Horikawa et al..$^{21}$ and Turnquist et al., ${ }^{20}$ and its specificity to $\Delta 133 p 53$ has been established in these previous publications. Commercially available primary antibodies used were as follows: anti-Oct-4 (sc-5279, Santa Cruz Biotechnology, Dallas, TX, USA; 1:1000); anti-p53 (DO-1, Santa Cruz Biotechnology; 1:2000); antip53 (PAb421, EMD Millipore, Billerica, MA, USA; 1:1000); anti-p62/SQSTM1 (PM045, MBL International, Woburn, MA, USA; 1:5000); anti-LC3B (\#2775, Cell Signaling Technology, Danvers, MA, USA; 1:2000); anti-Beclin-1 (\#3788, Cell Signaling Technology; 1:2000); anti-ATG7 (PM039, MBL International; 1:5000); antiATG5 (\#2630, Cell Signaling Technology; 1:5000); anti-GAPDH (EMD Millipore; 1:5000) and anti- $\beta$-actin (AC-15, Sigma-Aldrich; 1:10 000).

RNA isolation and qRT-PCR. Total RNA samples were prepared using TRIzol (Thermo Fisher Scientific). Reverse transcription was carried out using SuperScript III 1st strand CDNA synthesis system (Thermo Fisher Scientific). Quantitative real-time RT-PCR (qRT-PCR) assays were performed using Taqman Universal PCR Master Mix (Thermo Fisher Scientific) and the following primers/probe sets (all from Thermo Fisher Scientific): p21 ${ }^{\text {WAF1 }}$ (CDKN1A, Hs99999142_m1); PAl-1 (SERPINE1, Hs01126606_m1); IGFBP7 (Hs00944483_m1); BAX (Hs00180269_m1); PUMA (BBC3, Hs00248075_m1); p53R2 (RRM2B, Hs00968432_m1); and Nanog (Hs02387400_g1). GAPDH (Hs03929097_g1) was used as an internal control. For miR-34a, reverse transcription was performed using Taqman MicroRNA Reverse Transcription Kit and a miR-34a-specific primer, followed by quantitative real-time PCR reaction using Taqman MicroRNA Assay for miR-34a. RNU66 was used as a control for miR-34a (all reagents were from Thermo Fisher Scientific). Quantitative data analysis was performed using the $\Delta \Delta \mathrm{Ct}$ method according to the supplier's protocol (http://www3.appliedbiosystems.com/cms/groups/mcb_support/documents/generaldocuments/cms_040980.pdf). All qRT-PCR data were means \pm S.D. from technical triplicate $(n=3)$. The qRT-PCR experiments for the effect of $\Delta 133 \mathrm{p} 53$ on p53inducible genes were replicated in two independent fibroblast strains BJ (Figure 3) and CRL-2097 (Supplementary Figure S2). The conventional RT-PCR analysis shown in Supplementary Figure S1b used the following primers: $5^{\prime}-T G G$ GTT GCA GGA GGT GCT TAC-3' and 5'-CCA CTC GGA TAA GAT GCT GAG G-3' for $\triangle 133$ p53 (32 cycles); and 5'-CCA TCT TCC AGG AGC GAG A-3' and 5'-TGT CAT ACC AGG AAA TGA GC-3' for GAPDH (17 cycles). Quantitative image analysis was performed using the ImageJ $1.40 \mathrm{~g}$ software (http://rsb.info.nih.gov/ij).

Vectors for $\Delta 133 p 53$ overexpression and p53 knockdown and their transduction. A retroviral shRNA construct for p53 knockdown, which targets amino acid positions 259-264, was derived from pSUPER.retro.puro (OligoEngine, Seattle, WA, USA). ${ }^{18}$ A control construct containing the scrambled sequence was also generated from this vector. A retroviral construct for $\Delta 133 p 53$ overexpression was generated by inserting the $\triangle 133 \mathrm{p} 53 \mathrm{cDNA}$ into $\mathrm{pQCXIN}$ vector (BD Biosciences). ${ }^{18}$ The $\triangle 133$ p53 cDNA was also cloned into pLenti6.3/TO/V5DEST vector (Thermo Fisher Scientific) by using Spe I and Mlu I sites. This lentiviral $\Delta 133 p 53$ expression vector was used for either constitutive overexpression by itself (in Figures $4 c$ and 6 and Supplementary Figure S2) or inducible overexpression with a Tet repressor vector pLenti3.3/TR (Thermo Fisher Scientific) (in Figure $4 d$ and Supplementary Figure S4b). These retroviral or lentiviral vectors were transiently transfected into 293T/17 cells (ATCC) with the ViraPort retroviral expression system (Agilent Technologies, Santa Clara, CA, USA) or the ViraPower lentiviral expression system (Thermo Fisher Scientific), respectively. Vector supernatants were collected $48 \mathrm{~h}$ after transfection and then used to transduce human fibroblasts for $16-20 \mathrm{~h}$. Two days after transduction, the cells were selected with puromycin ( $2 \mu \mathrm{g} / \mathrm{ml}$; Sigma-Aldrich), G418 (500 $\mu \mathrm{g} / \mathrm{ml}$; Sigma-Aldrich) or blasticidin ( $5 \mu \mathrm{g} / \mathrm{ml}$; Thermo Fisher Scientific). Knockdown of full-length p53 without affecting $\triangle 133 p 53$ was performed by Lipofectamine RNAiMAX (Thermo Fisher Scientific)-mediated transfection of a siRNA oligonucleotide targeting amino acid positions 49-54 (Thermo Fisher Scientific, ID\# 115182), along with a negative control oligonucleotide (Thermo Fisher Scientific, cat\# AM4611).

iPSC reprogramming via retroviral vector transduction. Retroviral vector-mediated iPSC reprogramming was carried out as previously described. ${ }^{32}$ Briefly, vector supernatants were prepared from four retroviral pMXs constructs driving each of the Yamanaka reprogramming factors (i.e., Oct-4, Sox-2, Klf-4 and c-Myc), ${ }^{55}$ as described above. Human fibroblasts, which had been retrovirally or lentivirally transduced and selected with appropriate antibiotics as above, were plated in six-well plates $\left(2 \times 10^{5}\right.$ cells per well), and then infected with either all of the four vector supernatants or three of them (Oct-4, Sox-2 and Klf-4) every $12 \mathrm{~h}$ for 2 days. Three days after the completion of vector infection, the cells were harvested 
and replated on SNL feeder cells, followed by medium replacement with the above DMEM/F12-20\% KSR medium. Culture was maintained for 3-4 weeks in the absence of drug selection with daily medium changes. After several well-separated iPSC colonies were picked up for expansion, all the plates were stained for alkaline phosphatase (AP) using AP Staining Kit II (Stemgent, Lexington, MA, USA). For quantitation of iPSC generation efficiency, each reprogramming transduction had a parallel transduction containing the same set of reprogramming vectors plus a GFP-expressing vector (pBabe-Puro-GFP). ${ }^{32}$ The transduction efficiency was measured by flow cytometry detection of GFP and used to normalize data from the total number of AP staining-positive colonies. Data were means \pm S.D. from biological triplicate $(n=3$; three independent vector transductions of the Yamanaka reprogramming factors).

iPSC reprogramming via synthetic mRNA transfection. Reprogramming by means of transfection of synthetic modified mRNAs ${ }^{33}$ was carried out using the mRNA Reprogramming Factors Set (Stemgent) according to the supplier's protocol (http://assets.stemgent.com/files/1310/original/Stemgent_mRNAUserManual_2012.pdf). Human fibroblasts were plated on NuFF-1 MITC feeder cells (GlobalStem, Rockville, MD) in 6-well plates at $2.5 \times 10^{4}$ cells per well (for picking up colonies) or at $5 \times 10^{4}$ cells per well (for AP staining). Starting from the following day, mRNA transfection was performed using Lipofectamine RNAiMAX (Thermo Fisher Scientific) daily for 18 consecutive days, the first 6 days of which used fresh Pluriton mRNA Reprogramming Medium (Stemgent) and the remaining 12 days with the same medium pre-incubated with NuFF-1 MITC feeder. Each mRNA transfection consisted of recommended amounts of mRNAs encoding the four Yamanaka reprogramming factors (Oct-4, Sox-2, Klf-4 and c-Myc) and an mRNA encoding nuclear GFP, which allowed the confirmation of similar transfection efficiencies between control and $\Delta 133 p 53$-overexpressing cells. After 3-5 more days of daily medium changes with NuFF-1 MITC-conditioned Pluriton medium, visible colonies were counted on AP-stained and unstained wells. Data were means \pm S.D. from biological triplicate $(n=3$; three independent transfections of the synthetic mRNA cocktail). After colony counting, well-separated colonies were isolated from unstained wells for expansion.

Teratoma formation assay. Immunodeficient NOD/SCID mice were purchased from Charles River Laboratory (Frederick, MD, USA). IPSC in culture were treated with Y-27632 (Sigma-Aldrich) at $10 \mu \mathrm{M}$ for $3 \mathrm{~h}$ and harvested into Primate ES Cell Culture Medium (ReproCELL) with $10 \mu \mathrm{M}$ of Y-27632. The iPSC colonies were broken into small clumps by pipetting up and down several times. Per injection site, approximately $4 \times 10^{6}$ cells were suspended in $200 \mu$ l of the above medium containing 50\% Matrigel (BD Biosciences), ${ }^{56}$ followed by subcutaneous injection into the dorsal flank of two NOD/SCID mice per iPSC line. When cystic masses grew rapidly, cystic fluid was drained with a sterile syringe so that solid parts would grow for several days ${ }^{56}$ before mice were euthanized at 7 weeks after injection. When solid masses grew slowly, mice were maintained through 21 weeks after injection. All animal experiments and maintenance conformed to the guidelines of the Animal Care and Use Committee and of the American Association of Laboratory Animal Care. Resected teratoma tissues were fixed in 10\% formalin, embedded in paraffin blocks, sectioned at a thickness of $5 \mu \mathrm{m}$, and mounted on glass slides. Staining with hematoxylin and eosin was performed using standard procedures.

Chromosome analysis. High-quality G-band karyotyping analysis was performed at Cell Line Genetics (Madison, WI, USA). Twenty metaphases were analyzed per cell line and the results were verified by clinically certified cytogeneticists. When all 20 metaphases examined were normal or when at least 18 metaphases were normal with one or two demonstrating a non-clonal, simple chromosome loss (which is most likely a technical artifact), the cell line was concluded to have normal karyotype.

Isolation of genomic DNA. Genomic DNA samples were purified from cultured fibroblasts and PSC lines using PureLink Genomic DNA Mini kit (Thermo Fisher Scientific), which was previously used to quantitate mitochondrial DNA copy numbers. ${ }^{57}$ The quantity and quality of DNA samples were verified by $1.0 \%$ agarose gel electrophoresis.

Whole-exome sequencing. Whole-exome sequencing was performed at Novogene Corporation (Chula Vista, CA, USA). One $\mu \mathrm{g}$ of genomic DNA per sample was used as input material for DNA sample preparation. Sequencing libraries were generated using Agilent SureSelect Human All Exon kit (Agilent Technologies) following manufacturer's recommendations and index codes were added to each sample. Briefly, fragmentation was carried out by the hydrodynamic shearing system Covaris S2 (Covaris, MA, USA) to generate 180- to 280- bp fragments. Remaining overhangs were converted into blunt ends via exonuclease/ polymerase activities and then the enzymes were removed. After adenylation of $3^{\prime}$-ends of DNA fragments, adapter oligonucleotides were ligated. DNA fragments with ligated adapter molecules on both ends were selectively enriched in a PCR reaction. After PCR reaction, they were hybridized with biotinylated probes, then streptavidin-coated magnetic beads were used to capture 334378 exons in 20965 genes. Captured libraries were PCR-amplified to add multiplex index tags. The products were purified using AMPure XP system (Beckman Coulter, Beverly, MA, USA) and quantified using the Agilent high-sensitivity DNA assay on Bioanalyzer 2100 system (Agilent Biotechnologies). The clustering of the index-coded samples was performed on a cBot Cluster Generation System using TruSeq PE Cluster Kit v4-cBot-HS (Illumia, San Diego, CA, USA) according to the manufacturer's instructions. After cluster generation, the library preparations were sequenced on an Illumina sequencing platform. Sequencing statistics of each sample are summarized in Supplementary Table S3. The somatic mutation calling algorithms Mutect and Strelka were used to detect somatic SNPs and InDels, respectively, in each iPSC line versus its original fibroblasts.

Microsatellite DNA analysis. Five unstable microsatellite loci (D5S107, D5S406, ACTC, D13S153 and BAT26) were PCR-amplified in a single multiplex reaction using the previously reported primer pairs (one primer of each pair was $5^{\prime}$-labeled with FAM) ${ }^{34}$ and Type-it Microsatellite PCR kit (Qiagen, Valencia, CA, USA) following the supplier's protocol. The PCR products, along with GeneScan 500 ROX Size Standard (Applied Biosystems, Foster City, CA, USA), were analyzed on a fluorescence-based capillary electrophoresis (3130xl Genetic Analyzer, Applied Biosystems).

Assay of mitochondrial DNA (mtDNA) copy number. The qRT-PCRbased quantitation of mtDNA copy numbers, in parallel to nuclear DNA copy numbers, was performed using $1.0 \mathrm{ng}$ of DNA samples and Human Mitochondrial DNA Copy Number Assay kit (Detroit R\&D, Detroit, MI, USA) following the supplier's protocol. The mtDNA copy numbers normalized to nuclear DNA copy numbers were calculated using the $\Delta \Delta \mathrm{Ct}$ method.

DNA sequencing of mtDNA. To examine whether the iPSC lines established in this study harbor homoplasmic or heteroplasmic mtDNA mutations, ${ }^{35}$ DNA samples from the iPSC lines and the original fibroblasts were PCR-amplified for a mtDNA D-loop region with frequent mutations (nt. 128-599) using the primers $5^{\prime}$-CTG TCT TTG ATT CCT GCC TC-3' and 5'-TTG AGG TAA GCT ACA T-3', as previously reported, ${ }^{58}$ and Platinum Taq DNA Polymerase High Fidelity (Thermo Fisher Scientific). The PCR products were purified through Performa DTR Gel Filtration Cartridges (EdgeBio, San Jose, CA, USA), followed by direct sequencing in both directions using BigDye Terminator v1.1 Cycle Sequencing kit (Thermo Fisher Scientific).

Chromatin immunoprecipitation (ChIP). Immortalized BJ fibroblasts with $\Delta 133 p 53$ overexpression or control vector were cultured in $10-\mathrm{cm}$ plates to $\sim 90 \%$ confluence, and one plate was used for each immunoprecipitation. Cells were treated with $50 \mu \mathrm{M}$ etoposide (Sigma-Aldrich) during the last $24 \mathrm{~h}$ of culture, followed by fixation with $1 \%$ formaldehyde (Sigma-Aldrich) for 15 min with gentle agitation and then treatment with $125 \mathrm{mM}$ glycine (Sigma-Aldrich) for $10 \mathrm{~min}$. Fixed cells were rinsed with phosphate-buffered saline (PBS) twice, collected into microtubes using cell scraper, and lysed with ChIP lysis buffer $(50 \mathrm{mM}$ Tris- $\mathrm{HCl}(\mathrm{pH}$ 8.0), $10 \mathrm{mM}$ EDTA, 1\% SDS, Complete Protease Inhibitor Cocktail (Roche Diagnostics)) on ice for $10 \mathrm{~min}$. To fragment chromatin, samples were sonicated using a Bioruptor (Diagenode, Denville, NJ) for 3 cycles of $30 \mathrm{~s}$ (output level High) with an interval of $30 \mathrm{~s}$. Then, samples were centrifuged at $14000 \times g$ at $4{ }^{\circ} \mathrm{C}$ for $10 \mathrm{~min}$ and supernatants were collected. After taking an aliquot for input control ( $5 \%$ of total volume), the remaining samples were diluted 10 -fold in ChIP dilution buffer (20 mM Tris- $\mathrm{HCl}$ (pH 8.0), $150 \mathrm{mM} \mathrm{NaCl}, 2 \mathrm{mM}$ EDTA, 1\% Triton X-100, Complete Protease Inhibitor Cocktail), and then incubated overnight at $4{ }^{\circ} \mathrm{C}$ with $40 \mu$ l of Dynabeads M-280 sheep anti-mouse IgG (Thermo Fisher Scientific), which had been pre-incubated for $3 \mathrm{~h}$ with $5 \mu \mathrm{g}$ of DO-1 antibody (sc-126X, Santa Cruz Biotechnology). After washed with ChIP wash buffer (50 mM HEPES-KOH (pH 7.0), $0.5 \mathrm{M} \mathrm{LiCl}, 1 \mathrm{mM}$ EDTA, $0.7 \%$ deoxycholate, $1 \% \mathrm{NP}-40$ ) five times and with TE 
(10 mM Tris- $\mathrm{HCl}(\mathrm{pH} 7.5), 1 \mathrm{mM}$ EDTA) once, immunoprecipitated materials were eluted from the beads by heating overnight at $65^{\circ} \mathrm{C}$ in elution buffer $(50 \mathrm{mM}$ Tris$\mathrm{HCl}(\mathrm{pH} 8.0), 10 \mathrm{mM}$ EDTA, 1\% SDS). From these immunoprecipitated materials and the above aliquot taken before immunoprecipitation, DNA was purified using MinElute PCR purification kit (Qiagen). Quantitative PCR (qPCR) was performed using PowerUp SYBR Green Master Mix (Thermo Fisher Scientific) and the following primers: $\mathrm{HBB}$ (hemoglobin subunit $\beta$ as a negative control locus), 5'-AAC GTG CTC GCC TTT CTC-3' (forward) and 5'-GAA GCA GAACTC TGC ACT TC-3' (reverse); p21 WAF1, 5'-AGC CTT CCT CAC ATC CTC CT-3' (forward) and 5'-GGA ATG GTG AAA GGT GGA AA-3' (reverse); miR-34a, 5'-GGC ACG AGC AGG AAG GAG-3' (forward) and 5'-AAT CTC CAA ATG CCC CCG AT-3' (reverse); BAX, 5'-AGG CTG AGA CGG GGT TAT CT-3' (forward) and 5'-AAA GCT CAG AGG CCC AAA AT-3' (reverse); and PUMA, 5'-GTC GGTC TG TGTACG CAT CG-3' (forward) and 5'-AGA CAC CGG GAC AGT CGG ACA C-3' (reverse). DNA from the aliquot before immunoprecipitation was serially diluted and used to generate a standard curve for quantitative determination of DNA binding data calculated as $\%$ input. Data were means \pm S.D. from biological triplicate ( $n=3$; three independent 10-cm plates for immunoprecipitation).

Statistical analysis. Data are presented as means \pm S.D. from triplicate experiments $(n=3)$ unless otherwise stated. Statistical comparisons were made using unpaired two-tailed Student's $t$ test. Differences were considered significant at a value of ${ }^{*} P<0.05,{ }^{* *} P<0.01,{ }^{* * *} P<0.001$ or ${ }^{* * * *} P<0.0001$.

\section{Conflict of Interest}

The authors declare no conflict of interest.

Acknowledgements. We thank Michael Tainsky for cells, Elisa Spillare for continuous support, Valery Bliskovsky for valuable advice, Evgeny Arons and Steven Shema for microsatellite assay. This research was supported by the Intramural Research Program of the $\mathrm{NIH}, \mathrm{NCl}$. Capillary electrophoresis of microsatellite and sequencing reactions was conducted at the $\mathrm{CCR}$ Genomics Core at $\mathrm{NCl}$.

\section{Author contributions}

$\mathrm{IH}, \mathrm{KYP}, \mathrm{KI}, \mathrm{MS}$ and $\mathrm{CCH}$ conceived and designed the project. IH, KYP, $\mathrm{KI}, \mathrm{HL}, \mathrm{YH}$, $\mathrm{KA}$, AIR, AMM and KF performed the experiments. IH, KYP, KI, HL, YH, KA, AIR, MS and $\mathrm{CCH}$ analyzed the data. All authors contributed to writing the manuscript.

1. Bieging KT, Mello SS, Attardi LD. Unravelling mechanisms of p53-mediated tumour suppression. Nat Rev Cancer 2014; 14: 359-370.

2. Feng Z, Lin M, Wu R. The regulation of aging and longevity: a new and complex role of p53. Genes Cancer 2011; 2: 443-452.

3. Rodier F, Campisi J, Bhaumik D. Two faces of p53: aging and tumor suppression. Nucleic Acids Res 2007; 35: 7475-7484.

4. Singh SR. Stem cell niche in tissue homeostasis, aging and cancer. Curr Med Chem 2012; 19: 5965-5974.

5. Molchadsky A, Rivlin N, Brosh R, Rotter V, Sarig R. p53 is balancing development, differentiation and de-differentiation to assure cancer prevention. Carcinogenesis 2010; 31: $1501-1508$.

6. Tapia N, Scholer HR. p53 connects tumorigenesis and reprogramming to pluripotency. J Exp Med 2010; 207: 2045-2048.

7. Banito A, Rashid ST, Acosta JC, Li S, Pereira CF, Geti I et al. Senescence impairs successful reprogramming to pluripotent stem cells. Genes Dev 2009; 23: 2134-2139.

8. Hong H, Takahashi K, Ichisaka T, Aoi T, Kanagawa O, Nakagawa M et al. Suppression of induced pluripotent stem cell generation by the p53-p21 pathway. Nature 2009; 460: 1132-1135.

9. Krizhanovsky V, Lowe SW. Stem cells: the promises and perils of p53. Nature 2009; 460: 1085-1086.

10. Marion RM, Strati K, Li H, Murga M, Blanco R, Ortega S et al. A p53-mediated DNA damage response limits reprogramming to ensure iPS cell genomic integrity. Nature 2009; 460: 1149-1153.

11. Sarig R, Rivlin N, Brosh R, Bornstein C, Kamer I, Ezra O et al. Mutant p53 facilitates somatic cell reprogramming and augments the malignant potential of reprogrammed cells. J Exp Med 2010; 207: 2127-2140.

12. Mosteiro L, Pantoja C, Alcazar N, Marion RM, Chondronasiou D, Rovira M et al. Tissue damage and senescence provide critical signals for cellular reprogramming in vivo. Science 2016; 354: aaf4445.

13. Menendez S, Camus S, Izpisua Belmonte JC. p53: guardian of reprogramming. Cell Cycle 2010; 9: 3887-3891.
14. Zhao T, Xu Y. p53 and stem cells: new developments and new concerns. Trends Cell Biol 2010; 20: 170-175

15. Dannenmann B, Lehle S, Hildebrand DG, Kubler A, Grondona P, Schmid V et al. High glutathione and glutathione peroxidase-2 levels mediate cell-type-specific DNA damage protection in human induced pluripotent stem cells. Stem Cell Rep 2015; 4 : 886-898.

16. Qin H, Yu T, Qing T, Liu Y, Zhao Y, Cai J et al. Regulation of apoptosis and differentiation by p53 in human embryonic stem cells. J Biol Chem 2007; 282: 5842-5852.

17. Bourdon JC, Fernandes K, Murray-Zmijewski F, Liu G, Diot A, Xirodimas DP et al. p53 isoforms can regulate p53 transcriptional activity. Genes Dev 2005; 19: 2122-2137.

18. Fujita K, Mondal AM, Horikawa I, Nguyen GH, Kumamoto K, Sohn JJ et al. p53 isoforms $\Delta 133 p 53$ and $p 53 \beta$ are endogenous regulators of replicative cellular senescence. Nat Cell Biol 2009; 11: 1135-1142.

19. Mondal AM, Horikawa I, Pine SR, Fujita K, Morgan KM, Vera E et al. p53 isoforms regulate aging- and tumor-associated replicative senescence in T lymphocytes. J Clin Invest 2013; 123: $5247-5257$

20. Turnquist C, Horikawa I, Foran E, Major EO, Vojtesek B, Lane DP et al. p53 isoforms regulate astrocyte-mediated neuroprotection and neurodegeneration. Cell Death Differ 2016; 23 : 1515-1528.

21. Horikawa I, Fujita K, Jenkins LM, Hiyoshi Y, Mondal AM, Vojtesek B et al. Autophagic degradation of the inhibitory $p 53$ isoform $\Delta 133 p 53 \alpha$ as a regulatory mechanism for $p 53$ mediated senescence. Nat Commun 2014; 5: 4706

22. Shaid S, Brandts $\mathrm{CH}$, Serve H, Dikic I. Ubiquitination and selective autophagy. Cell Death Differ 2013; 20: 21-30.

23. Neveu P, Kye MJ, Qi S, Buchholz DE, Clegg DO, Sahin M et al. MicroRNA profiling reveals two distinct p53-related human pluripotent stem cell states. Cell Stem Cell 2010; 7 : 671-681.

24. Brown JP, Wei W, Sedivy JM. Bypass of senescence after disruption of $\mathrm{p} 21^{\mathrm{CIP} 1 / \text { WAF1 }}$ gene in normal diploid human fibroblasts. Science 1997; 277: 831-834.

25. Kortlever RM, Higgins PJ, Bernards R. Plasminogen activator inhibitor-1 is a critical downstream target of p53 in the induction of replicative senescence. Nat Cell Biol 2006; 8 : 877-884.

26. Severino V, Alessio N, Farina A, Sandomenico A, Cipollaro M, Peluso G et al. Insulin-like growth factor binding proteins 4 and 7 released by senescent cells promote premature senescence in mesenchymal stem cells. Cell Death Dis 2013; 4: e911.

27. Tazawa H, Tsuchiya N, Izumiya M, Nakagama H. Tumor-suppressive miR-34a induces senescence-like growth arrest through modulation of the E2F pathway in human colon cancer cells. Proc Natl Acad Sci USA 2007; 104: 15472-15477.

28. Miyashita T, Reed JC. Tumor suppressor $p 53$ is a direct transcriptional activator of the human bax gene. Cell 1995; 80: 293-299.

29. Nakano K, Vousden KH. PUMA, a novel proapoptotic gene, is induced by p53. Mol Cell2001; 7: 683-694.

30. Bourdon A, Minai L, Serre V, Jais JP, Sarzi E, Aubert S et al. Mutation of RRM2B, encoding p53-controlled ribonucleotide reductase (p53R2), causes severe mitochondrial DNA depletion. Nat Genet 2007; 39: 776-780.

31. Tanaka H, Arakawa H, Yamaguchi T, Shiraishi K, Fukuda S, Matsui K et al. A ribonucleotide reductase gene involved in a p53-dependent cell-cycle checkpoint for DNA damage. Nature 2000; 404: 42-49.

32. Li H, Collado M, Villasante A, Strati K, Ortega S, Canamero M et al. The Ink4/Arf locus is a barrier for iPS cell reprogramming. Nature 2009; 460: 1136-1139.

33. Warren L, Manos PD, Ahfeldt T, Loh YH, Li H, Lau F et al. Highly efficient reprogramming to pluripotency and directed differentiation of human cells with synthetic modified mRNA. Cell Stem Cell 2010; 7: 618-630.

34. Sutter C, Gebert J, Bischoff P, Herfarth C, von Knebel Doeberitz M. Molecular screening of potential HNPCC patients using a multiplex microsatellite PCR system. Mol Cell Probes 1999; 13: 157-165.

35. Prigione A, Lichtner B, Kuhl H, Struys EA, Wamelink M, Lehrach $\mathrm{H}$ et al. Human induced pluripotent stem cells harbor homoplasmic and heteroplasmic mitochondrial DNA mutations while maintaining human embryonic stem cell-like metabolic reprogramming. Stem Cells 2011; 29: 1338-1348

36. Gore A, Li Z, Fung HL, Young JE, Agarwal S, Antosiewicz-Bourget J et al. Somatic coding mutations in human induced pluripotent stem cells. Nature 2011; 471: 63-67.

37. Ji J, Ng SH, Sharma V, Neculai D, Hussein S, Sam M et al. Elevated coding mutation rate during the reprogramming of human somatic cells into induced pluripotent stem cells. Stem Cells 2012; 30: 435-440.

38. Tang Y, Horikawa I, Ajiro M, Robles Al, Fujita K, Mondal AM et al. Downregulation of splicing factor SRSF3 induces $\mathrm{p} 53 \beta$, an alternatively spliced isoform of $\mathrm{p} 53$ that promotes cellular senescence. Oncogene 2013; 32: 2792-2798.

39. Marcel V, Vijayakumar V, Fernandez-Cuesta L, Hafsi H, Sagne C, Hautefeuille A et al. p53 regulates the transcription of its $\Delta 133 p 53$ isoform through specific response elements contained within the TP53 P2 internal promoter. Oncogene 2010; 29: 2691-2700.

40. Wei J, Noto J, Zaika E, Romero-Gallo J, Correa P, El-Rifai W et al. Pathogenic bacterium Helicobacter pylori alters the expression profile of p53 protein isoforms and p53 response to cellular stresses. Proc Natl Acad Sci USA 2012; 109: E2543-E2550.

41. Choi YJ, Lin CP, Ho JJ, He X, Okada N, Bu P et al. miR-34 miRNAs provide a barrier for somatic cell reprogramming. Nat Cell Biol 2011; 13: 1353-1360. 
42. Kawamura T, Suzuki J, Wang YV, Menendez S, Morera LB, Raya A et al. Linking the p53 tumour suppressor pathway to somatic cell reprogramming. Nature 2009; 460 : 1140-1144.

43. Rasmussen MA, Holst B, Tumer Z, Johnsen MG, Zhou S, Stummann TC et al. Transient p53 suppression increases reprogramming of human fibroblasts without affecting apoptosis and DNA damage. Stem Cell Rep 2014; 3: 404-413.

44. Momcilovic O, Knobloch L, Fornsaglio J, Varum S, Easley C, Schatten G. DNA damage responses in human induced pluripotent stem cells and embryonic stem cells. PLOS One 2010; 5: e13410.

45. Das S, Raj L, Zhao B, Kimura Y, Bernstein A, Aaronson SA et al. Hzf Determines cell survival upon genotoxic stress by modulating p53 transactivation. Cell 2007; 130: 624-637.

46. Llanos S, Cuadrado A, Serrano M. MSK2 inhibits p53 activity in the absence of stress. Sci Signal 2009; 2: ra57.

47. Tanaka T, Ohkubo S, Tatsuno I, Prives C. hCAS/CSE1L associates with chromatin and regulates expression of select p53 target genes. Cell 2007; 130: 638-650.

48. Kitayner M, Rozenberg H, Kessler N, Rabinovich D, Shaulov L, Haran TE et al. Structural basis of DNA recognition by p53 tetramers. Mol Cell 2006; 22: 741-753.

49. Ben-David U, Benvenisty N. The tumorigenicity of human embryonic and induced pluripotent stem cells. Nat Rev Cancer 2011; 11: 268-277.

50. Folmes CD, Terzic A. Energy metabolism in the acquisition and maintenance of stemness. Semin Cell Dev Biol 2016; 52: 68-75.
51. Pan H, Cai N, Li M, Liu GH, Izpisua Belmonte JC. Autophagic control of cell 'stemness'. EMBO Mol Med 2013; 5: 327-331.

52. Gong L, Gong H, Pan X, Chang C, Ou Z, Ye S et al. p53 isoform $\Delta 113 p 53 / \Delta 133 p 53$ promotes DNA double-strand break repair to protect cell from death and senescence in response to DNA damage. Cell Res 2015; 25: 351-369.

53. Nakamura AJ, Chiang YJ, Hathcock KS, Horikawa I, Sedelnikova OA, Hodes RJ et al. Both telomeric and non-telomeric DNA damage are determinants of mammalian cellular senescence. Epigenetics Chromatin 2008; 1 : 6.

54. Mallon BS, Chenoweth JG, Johnson KR, Hamilton RS, Tesar PJ, Yavatkar AS et al. StemCellDB: the human pluripotent stem cell database at the National Institutes of Health. Stem Cell Res 2013; 10: 57-66.

55. Takahashi K, Tanabe K, Ohnuki M, Narita M, Ichisaka T, Tomoda K et al. Induction of pluripotent stem cells from adult human fibroblasts by defined factors. Cell 2007; 131: 861-872.

56. Prokhorova TA, Harkness LM, Frandsen U, Ditzel N, Schroder HD, Burns JS et al. Teratoma formation by human embryonic stem cells is site dependent and enhanced by the presence of Matrigel. Stem Cells Dev 2009; 18: 47-54.

57. Maes H, Van Eygen S, Krysko DV, Vandenabeele P, Nys K, Rillaerts K et al. BNIP3 supports melanoma cell migration and vasculogenic mimicry by orchestrating the actin cytoskeleton. Cell Death Dis 2014; 5: e1127.

58. Yu M, Shi $Y$, Zhang F, Zhou Y, Yang $Y$, Wei $X$ et al. Sequence variations of mitochondrial DNA D-loop region are highly frequent events in familial breast cancer. J Biomed Sci 2008; 15: $535-543$.

\section{Supplementary Information accompanies this paper on Cell Death and Differentiation website (http://www.nature.com/cdd)}

